\title{
Alternate thematic maps to visualize United Nations Sustainable Development Goal indicator data
}

\author{
Natasha Pirani ${ }^{a}$, Britta Ricker ${ }^{a}$, Menno-Jan Kraak ${ }^{\mathrm{a}}$ \\ aUniversity of Twente; pirani36986@alumni.itc.nl; b.a.ricker@utwente.nl;m.j.kraak@utwente.nl \\ * Corresponding author
}

\begin{abstract}
The choropleth is a widely used thematic map type. But it is not always ideal to visualize social data in engaging and accurate ways, especially as a standalone map. In this paper we discuss choropleths and two thematic map types with altered geometry: area cartograms and tile maps with repeating icons. To identify benefits and drawbacks of each, we created a choropleth, contiguous cartogram, and repeating icon tile map visualizing the same data from the United Nations Sustainable Development Goal (SDG) indicator about the proportion of women and girls aged 15-49 who have undergone female genital mutilation/cutting in African countries, from SDG 5, on Gender Equality. We conducted a qualitative online survey to collect users' evaluations, through informational tasks, quality ratings, and open-ended questions based on interaction with the maps. Results of this preliminary investigation suggest that though users are familiar and therefore more comfortable with choropleth maps, they interpreted thematic map types differently. Specifically, the relative novelty and unfamiliarity of the distorted geometry of cartograms and tile maps may have caused users to engage more thoughtfully with the visualized data and in the cartogram and tile map which are generally considered non-standard thematic maps.
\end{abstract}

Keywords: thematic maps, Sustainable Development Goals, cartogram, tile map

\section{Introduction}

Thematic maps are useful tools to communicate sociogeographic information. The positivist, traditional approach to map design usually produces a single map, with one cartographic viewpoint - but multiple maps can promote informed scepticism, and foster more complete understanding (Monmonier, 1991) of data, phenomena, and how they have been mapped. The United Nations has aggregated and made available data by country at a global scale to measure progress towards reaching the interrelated Sustainable Development Goals (SDG) that address social inclusion, economic growth, and environmental protection (United Nations, 2018). Often, a choropleth is used to map statistical data (Crampton, 2009); for example, the World Bank's Visualize Inequality dashboard (The World Bank, 2018) maps country-level access to resources and services as choropleths, and Our World in Data's SDG Tracker (Roser, 2018) maps SDG indicator data as world choropleth maps.

The choropleth may not be the most communicative, accurate, or engaging thematic map type for every dataset, despite its wide use. For this research, we are interested to find how multiple and less familiar, alternative representations of space depicting the same data can evoke different perceptions in users, even if the maps may be met with initial discomfort. As Sui \& Holt (2008) write: "we should not underestimate the effects of inertia in map readers' adjustment to new views of their world" (p. 15). We first review qualities of choropleths and two other thematic map types with altered geometry: area cartograms and tile maps. To examine users' interpretations of less familiar geographies and map types, we conducted an online survey in which we gathered user feedback through informational tasks, open-ended questions, quality ratings, and demographics questions post-interaction with a choropleth, contiguous cartogram, and repeating icon tile map, each differently visualizing the same UN data.

The results show that users found the obviously altered geometry of the latter two map types unfamiliar and sometimes off-putting; some users were hesitant to embrace them, while some positive responses indicated curiosity and appreciation for the novelty. Mixed attitudes based on the combined novelty of these maps may have helped trigger deeper engagement with the presented SDG data than the traditional choropleth alone, even if users prefer one map type over another.

\section{Background}

\subsection{The Sustainable Development Goals (SDG)}

The United Nations SDG are seventeen universal, interrelated goals spanning social inclusion, economic growth, and environmental protection; they are international calls for action, to be implemented by each country. The goals are specified by 169 targets measured by 232 individual indicators in a global framework. They were adopted by world leaders in 2015 at the UN Sustainable Development Summit and came into effect on 1 January 2016 as the 2030 Agenda for Sustainable 
Development (United Nations, 2018; United Nations Statistics Division, 2018a).

SDG indicator data is available through the Global Database at the national scale, by country (United Nations Statistics Division, 2018). Completeness and timeliness vary by country and indicator. Some indicators are binary distinctions or parity indices, while many measure some proportion of a population, suitable for a classed choropleth map. We focused on SDG indicator 5.3.2, the proportion of women and girls aged 15-49 who have undergone female genital mutilation/cutting. This indicator is a measure of goal 5, to achieve gender equality and to empower all women and girls.

Female genital mutilation/cutting (FGM/C) is not well known or widely discussed in many societies, and its prevalence can be communicated to a wider audience through maps. FGM/C is the total or partial removal of or injury to the external female genital for non-medical reasons. It has been reported to occur all over the world. With no identified health benefits, it is known to be harmful, traumatic, and painful, with immediate and longterm health consequences. From human rights perspectives, the practice reflects ingrained gender inequality and extreme discrimination against women and children (World Health Organization, 2008).

\subsection{Multiple maps, multiple perspectives}

All information visualizations are subjective and open to interpretation; so too are all maps. Critical information visualization calls for plurality and the exposure of multiple perspectives to enable various interpretations and to challenge viewers to question their own assumptions (Dörk, Feng, Collins, \& Carpendale, 2013). In cartography, the traditional and positivist approach often results in a "one map solution" where a viewer is provided with a single, highly selective map that alone is insufficient to communicate the complexity of an issue and justification for the mapmaker's multiple decisions (Monmonier, 1991). Low "cartodiversity" in fields such as public health perpetuates the choropleth map as the standard thematic map type (Sui \& Holt, 2008, p. 4). But multiple thematic map types exist, which provide different viewpoints through altered spatial geometry and symbolization. Cognitive categories and definitions of maps are limited to the known, understood geopolitical world and its delineation of geographic space; alternate geometries, for example cartograms, show that space can be represented in different ways and that people can accommodate new map types (Huffman, 1997). In geovisualization, alternative visual representations of the same data in linked view environments stimulates visual thinking (Dykes. MacEachren \& Kraak, 2005).

\subsection{Thematic maps}

\subsubsection{Choropleth maps}

Choropleths are widely used, often to represent statistical data, and easier to produce and automate than alternatives (Crampton, 2004; Crampton, 2009). Each polygon usually represents a geopolitical area, and a choropleth may be misleading if it visualizes raw counts for a population- related variable rather than relative or standardised data as area and population are unrelated, it may not adequately portray the spatial pattern of the issue. Standardizing raw counts by a variable such as population does not make it possible to distinguish between areas with high incidence and high population or areas with low incidence and low population (Roth, Woodruff, \& Johnson, 2010). Choropleths do not necessarily reflect real conditions or rates in a particular area (Crampton, 2004). Of course, if data, such as the SDG indicator data which are available by country, are collected and defined according to specific political boundaries, it is not always possibile to disaggregate it and reconstruct borders. But polygons can be redrawn to represent population or another variable, in the case of a cartogram - or to disregard difference and give each polygon equal space - in the case of a tile map. We will now review these two alternatives.

\subsubsection{Area cartograms}

An area cartogram can convey more information through deliberate distortion than a traditional choropleth map (Döll, 2017; Dorling et al., 2006) and in human geography some mapmakers consider cartograms to be a more socially just form of mapping because they provide a more equitable representation of the world (Dorling, 1996). Each input polygon is usually an administrative area that is rescaled proportionally to a related equalizing variable, such as population. Another statistic of interest can then be shown in a choropleth (colour shading) layer atop the distorted polygons and does not require standardization. A cartogram thus shows both statistical and geographical information from which the user may gain insight into outliers, patterns, and trends. Well-suited for visualizing political and socio-economic data, cartograms appear often in media, textbooks, and blogs, and are increasingly popular in other social, political, and public health applications (Nusrat \& Kobourov, 2016; Roth et al., 2010). Using a population or subpopulation value to resize each polygon can yield a demographic base map. Or the original map can be redrawn so that each unit is proportional to the variable of interest, which is effective if polygons are still identifiable. Users' previous experience and knowledge of the area and the phenomenon may limit a contiguous cartogram's utility (Monmonier, 1977) but users with limited cartogram experience in general may find the map interesting to read (Sui \& Holt, 2008).

There are several types of area cartograms. For each type there are many algorithms to produce different results; none is ideal, because in distorting area, all cartograms compromise one or more of statistical, geographical, and topological accuracy (Nusrat \& Kobourov, 2016). Contiguous cartograms distort area but retain adjacencies (topology). These cartograms maintain statistical, topological, and geographical accuracy better than many other types (Nusrat \& Kobourov, 2016). One of the most popular algorithms to create contiguous cartograms is by Gastner and Newman (2004); their algorithm uses a linear diffusion process to equalize population density across the cartogram, preserving topology and shape recognizability. Software is available to generate these cartograms (Nusrat \& Kobourov, 2016). The Worldmapper project, for 
example, has produced over six hundred cartograms of the world using the Gastner-Newman method, visualizing topics social and economic data on topics related to pollution, poverty, and education, and more (Hennig, Pritchard, Ramsden, \& Dorling, 2010). To represent every country on the cartogram, missing values are estimated, or in the case of very small territories, omitted (Worldmapper, 2018; Dorling et al., 2006).

Non-contiguous cartograms maintain statistical accuracy and shape, but not adjacency. Each undistorted polygon is independently resized, resulting in discrete polygons with gaps between them that renders recognition relatively uncomplicated for the user (Nusrat \& Kobourov, 2016; Olson, 1976). The size of any enumeration unit does not relate directly to the region's data, but depends on the data distribution across all regions. Some regions may become too small and unrecognizable, and the resulting "sparseness" or lack of contiguity and map feel may render a non-contiguous cartogram unpreferable, or difficult to interpret or comprehend. In a quantitative study of cartogram effectiveness based on users task performance (time and error), non-contiguous cartograms were found to be effective but users did not appreciate them as much (Nusrat, Alam, \& Kobourov, 2018).

Dorling (1996) created an algorithm to make circular cartograms, which abstract each polygon to a circle, the size of which represents the data value. The circles may touch, but not overlap, and shift if necessary, foregoing shape and accurate topology, but with potentially zero cartographic error. They are popular on the web, especially through d3 implementations (Nusrat et al., 2018; Nusrat \& Kobourov, 2016). Demers cartograms show squares instead of circles, so there are fewer gaps between regions. While Dorling cartograms keep regions as close to their original location as possible, Demers cartograms often forego distance to preserve continuity between regions and visual cues (Demers \& Bortins, 2002).

Mosaic cartograms are configurations of multiple, congruent square, triangular, or hexagonal tiles connected at the edges. They have become popular in the media to show electoral and demographic data in the US and UK (Cano et al., 2015). These cartograms often maintain a schematized version of the input polygon's shapes, and allow users to accurately compare regions. The configuration of tiles represents the region, within which individual tiles represent the data. So these maps require data that can be cast as small integer units, such as number of votes or people (Cano et al., 2015), but many SDG indicators are not absolute values.

\subsubsection{Tile maps and repeating icons}

Tile maps abstract each polygon to a single geometric tile in a configuration that retains local topology, global position, and global shape where possible, and thus resembles the original map shape and the user's mental model. Each tile is congruent so geographically large areas do not dominate and smaller areas are equally visible. Task-specific issues and previous knowledge influence how users perceive geographic features and relationships; user studies will help understand how adjacency, relative orientation, global shape, and other properties relate to human judgements of the accuracy of tile maps, which will also depend on personal preferences and prior knowledge and experiences (Mcneill \& Hale, 2017).

Tile maps tend to be reused, which indicates that they are in demand but not easy to make; several blog posts and websites discuss how to handcraft tile maps with tutorials for how to create them in Excel, Tableau, or d3 (e.g. DeBelius (2015)) rather than with an automated procedure or tool. Thus their uptake may be limited by the timeconsuming act of making them (Mcneill \& Hale, 2017).

Tile maps avoid the visual imbalance of choropleth maps and favour clarity over complexity when compared to cartograms (DeBelius, 2015). Various techniques can be used to show data on the tiles, such as choropleth shading, symbols, or charts. We focus on repeating icons in an Isotype-esque style; this technique avoids the perceptual noise some other symbols create, such as overlapping graduated circles (Monmonier, 1977).

In the 1920s Otto and Marie Neurath and Gerd Arntz created an international picture language called Isotype (International System Of TYpographic Picture Education) to visually represent social facts to uneducated and working-class populations. The aim was to provide a visible, tangible, and easily and universally understandable form to political and social ideas (Blau, 2006; Haroz, Kosara, \& Franconeri, 2015). These pictorial statistics used simple, minimally coloured symbols or icons as units representing fixed quantities, repeated in specific configurations (Neurath, 1974; Haroz et al., 2015). In combination with a tile map, a repeating pictorial icon can represent classified data in a novel, embodied way. For example, each symbol may represent five percent of the affected population, and half or other fraction of the full symbol may represent a smaller value. Each symbol is otherwise congruent and repeats in rows and columns, where the polygon fills up with $100 \%$ (in this example, 20 tiles) according to the value of the statistic.

\subsection{Related work}

One evaluation of cartogram effectiveness through users' performance and attitudes showed that contiguous Gastner-Newman cartograms perform well on metricsand task-based evaluations and by user preferences compared to other cartogram types (Nusrat et al., 2018). Sui and Holt (2008) also found Gastner-Newman cartograms to be evaluated cartograms through a framework that synthesizes three paradigms: cognitive (maps as images), analytical (maps as models or computational tools), and critical (maps as social constructs). The authors believe that cartograms heighten users' sensitivity to critical aspects of maps and mapmaking, reminding users that maps are consciously human made. Map readers with no prior knowledge of cartograms found them unusual, less helpful and less valuable, but an interesting way to visualize spatial information. Users were motivated to probe the cartograms' meaning because of their different appearance and readability. 
Gilmartin (1978) found users can respond subjectively to thematic maps using semantic differential (SD) scales which are sensitive to differences in design characteristics between maps. In the SD technique, a given scale ranges from one adjective (e.g. "bad", at -3 on one end) to its opposite (e.g. "good", at +3 ), with neutral in the middle. On or between these poles the user marks a rating reflecting their attitude toward the map in question (Gilmartin, 1978). Dent (1975) used SD scales, to test reader attitudes and responses towards area cartograms, e.g. "good/bad", "innovative/conventional", and "easy to $\mathrm{read} /$ difficult to read" (p. 163). Nusrat et al. (2018) used this system in their thorough evaluation of the effectiveness of cartograms to measure attitudes. Sui \& Holt (2008) use a similar process in their cognitive evaluation of cartograms. We too adapted the SD technique for our survey.

Haroz, Kosara, and Franconeri (2015) found that pictographs invite readers to look at a visualization more closely and can help them retain information during challenging tasks.

We have not found any user research on tile maps.

\section{User study}

\subsection{Survey design}

To address the objective of finding how multiple alternative representations of space depicting the same data can evoke different subjective evaluations in users, we conducted an online survey in which users were presented with a choropleth, contiguous cartogram, and repeating icon tile map in random order, each visualizing the same data about FGM/C. The study opened with an introduction about the survey, an overview of the map types with a sample of each, and an open question to gauge users' prior awareness of FGM/C. Each map was accompanied by two informational multiple-choice tasks, developed based on the taxonomy of cartogram tasks from Nusrat and Kobourov (2015): one locate and identify task, and one "find top-k" (find the highest value) task. For example: What is the percentage of women and girls aged 15-49 who have undergone FGM/C in Egypt? This task required locating the country and interpreting its corresponding attribute. "Which of the following countries has the highest proportion of women and girls aged 15-49 who has undergone FGM/C?" required locating the countries and determining which had the highest value of the statistic. Users were asked to complete the tasks after taking a minute to observe the map. After engaging with all maps, on a separate page the users rated the following qualities of each map on a five-point scale from "strongly disagree" to "strongly agree" with the mid-point as "neutral": interesting, truthful, symbolizes information well, shows relative values clearly, easy to understand, emotional. They were asked to supplement these ratings with comments. They could also comment specifically about questions that arose from the maps. Users were also asked to provide demographic information at the end of the survey, including age, gender, nationality, country of residence, occupation, and experience in a geo-related field. We thematically coded comments with keywords or topics that emerged from the responses themselves.

We report only on the results of the above-mentioned parts of the survey. The original also included four sets of selfreport questions about users' affective states before and after engaging with each map, and an open question about emotions experienced during the study.

\subsection{Thematic maps for the user survey}

We created and tested a choropleth, a contiguous cartogram, and a repeating icon tile map of the SDG indicator about the proportion of women and girls aged 1549 who have undergone FGM/C (see Figure 1). The data are available for 29 countries, 27 of which are in Africa, each country with one data point between 2004 and 2016. We mapped the data for African countries.

The cartogram and tile map challenge the choropleth conceptions of geographic space. The use of a repeating pictorial icon, or symbol can embody the data compared to the abstract choropleth use of colour. We adapted a scissors symbol from the Maki icon set (Mapbox, 2018). This symbol draws attention to the issue represented by the numbers and connotes injury. The use of repeating icons to represent classified data is inspired by the "tile grid waffle chart" web map (Schwabish, 2017) from the ONE (2018) campaign which represents African countries as square tiles and each percentage point of the visualized statistic as a smaller square tile that comprises one percent of the larger tile.

Contiguous Gastner-Newman cartograms fare relatively well by users' preferences and task performance (Nusrat et al., 2018), so we used this type of cartogram. In our map the size of each country has been re-sized according to population, rather than the statistical data, because the former is available for all countries while the latter was not. Absolute data for the indicators is also not available because they represent a proportion of a sub-population.

\subsection{Participants}

A convenience sample was used. An email was sent to some students and faculty from the University of Twente's Faculty of Geo-information Science and Earth Observation, the Erasmus Mundus Cartography MSc program, and to selected International Cartographic Association commissions. The survey link was also tweeted by the second author.

\subsection{Results and discussion}

55 people completed the study. All had used choropleth maps before, $87 \%$ had used cartograms, and $41 \%$ had used tile maps. 43 mentioned higher education or work in a georelated field, such as GIS and cartography.

Despite geographical distortions in the tile map (area shape, size, and topology), the average success rate across the two informational tasks was highest for this map type, at $97 \%$ (compared to $94 \%$ for both the choropleth and cartogram). Perhaps the time and extra effort required to read the map (based on users' comments) resulted in more care taken when completing the tasks. One said, "this map reminds of a data list, where the numbers are placed in a 

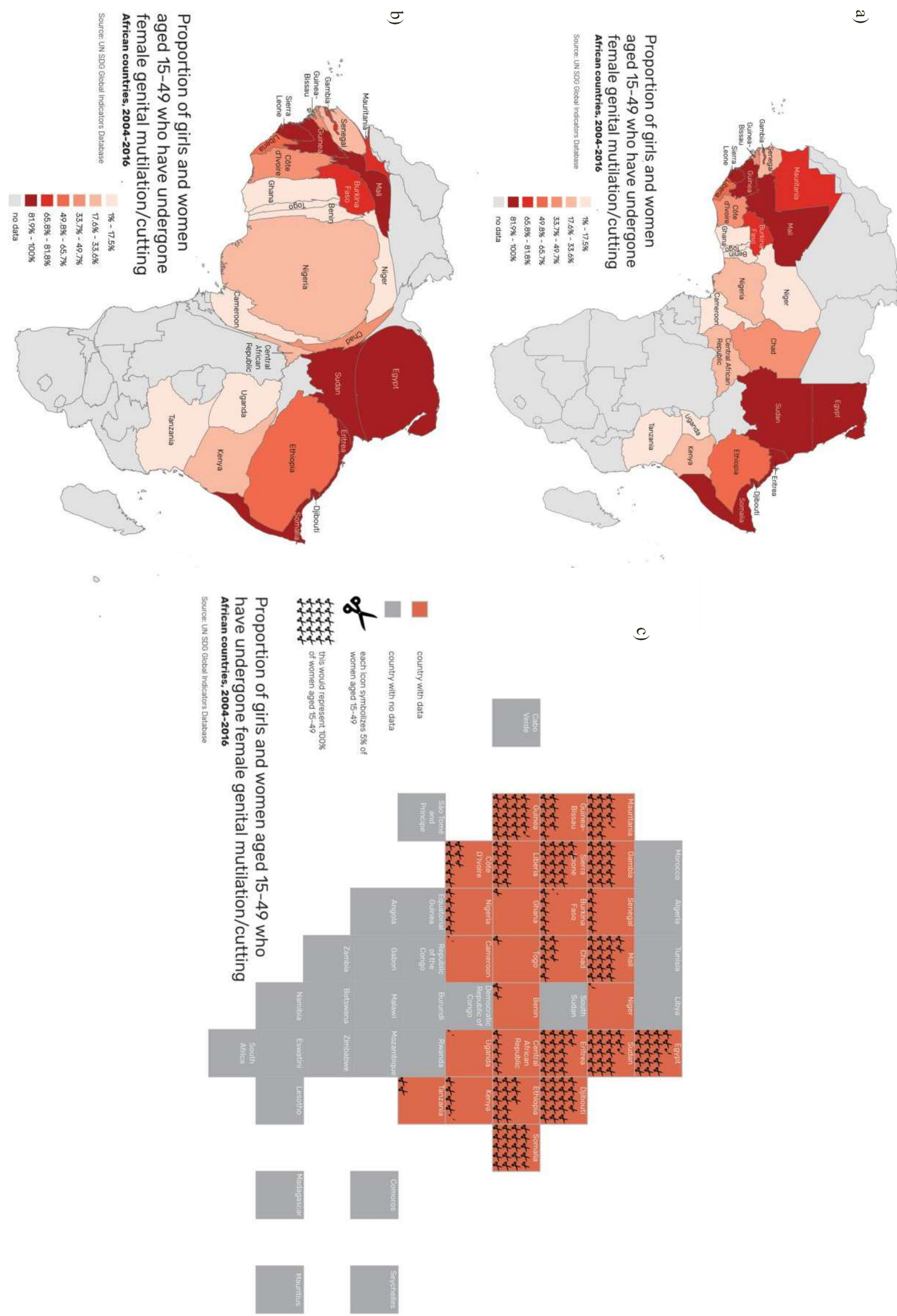

Figure 1. Thematic maps shown to users in the survey, visualizing the proportion of women and girls aged 15-49 who have undergone female genital mutilation/cutting, in African countries: a) choropleth; b) contiguous cartogram; c) tile map with repeating icon. Users saw these maps separately, in random order. 
somehow geographical way" (Participant 20), implying the information was organized but geography was not the priority. Survey responses revealed that the choropleth map was the easiest to understand but the least interesting, and least emotional. The cartogram was rated highest for "interesting" and "emotional", followed by the tile map was second (see Figure 2).

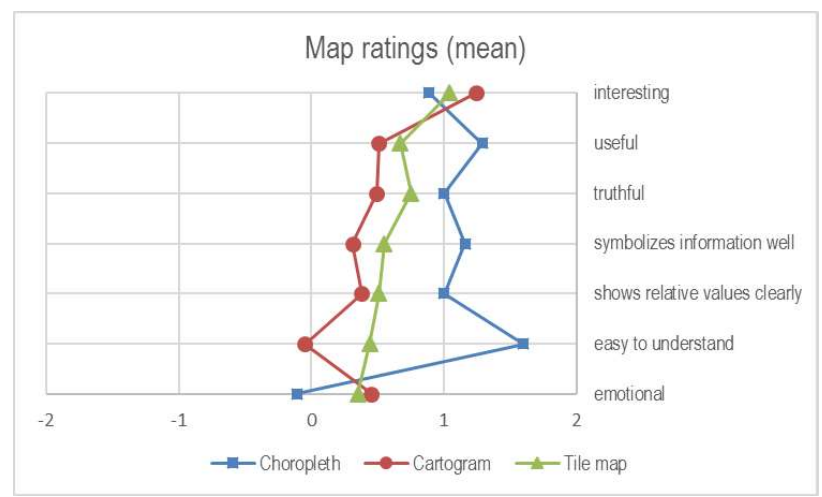

Figure 2. Mean ratings: choropleth, cartogram, and tile map.

There were 24 responses to open questions on the choropleth map, 27 responses to the cartogram, and 28 responses for the tile map. Eight of the comments on the choropleth map mentioned that it was easy to use; often this was linked to its familiarity or wide use: "simple, seen so many times before, that's why one probably doesn't spend too much time looking at it" (Participant 31); "Easiest type of map to understand due to its use frequency in media" (Participant 46).

Overall, the choropleth seemed to stimulate reflection on the usability of the map more than the design decisions or the issue of FGM/C itself, but some did acknowledge a limitation of the map: "it is easier to use and find countries, also it is easy to recognize the values based on color. However, it distorts the proportion, as it appears more women are affected by the larger surface area of large countries" (Participant 51). Three people said the choropleth was boring, a word which did not appear for the tile map nor cartogram. Its boringness is perhaps attributed to its familiarity and wide use.

Together, thematically coded mixed, neutral, and negative responses outweighed the positive responses to the cartogram. The four negative responses to the cartograms hinged vaguely on their unusual appearance and distortion: "they show the polygons in an unfamiliar way" (Participant 6); "looks like an inflamed pancreas" (Participant 23).

Positive responses indicated the cartogram showed more information or was easy to understand: "It provides the population as well as the statistic. Most informative map that shows quality as well as quantity" (Participant 4). Although the study briefly explained that the countries were distorted by population, some still thought the map showed the same statistic twice, through both distortion and colour: "here, the relative size of the countries is used to show the same information as with the colours. By that, the message is doubled/duplicated" (Participant 20), while one asked, "Why is the same topic visualised twice?!?" (Participant 50). Others suggested the cartogram would be more evocative if the variable, rather than population, was used to rescale countries. Were this data available for all countries, a very different map could be made, which would allow for shading or another technique to increase the complexity of the visualization through another layer. Negativity about the cartogram suggests that an unfamiliar map can provoke critique of the map, which may widen perspectives of the map, especially when in combination with other thematic maps. Nonetheless the cartogram did was not explicitly novel (as the tile map was), but aroused some curiosity: "so far quite an unusual display, thus raises potentially some interest" (Participant 10).

Several commented that the tile map was a neutral representation of the data: "it gives equal weight to all countries and includes a neutral representation of the rate" or "seems more neutral in some ways than the other two" (Participant 22) - perhaps due to the abstracted and simplified geometry (squares, to represent each country). Several found it unfamiliar, requiring more time and effort to use, which could be either a hindrance or an invitation to engage further: "Different map not very common. Not very good. Requires more attention and reading to understand the data" (Participant 21).

Generally, users did not find the tile map as easy to read, but that it required more time and a closer look: "it took quite some time to read the legend, to read all the tiles, and then to see" (Participant 48). The eight usability-themed comments (on how participants felt about its difficulty or the time needed to peruse it - effectiveness or efficiency) mentioned needing more time, a closer look, or that the map was hard to read. One mentioned it was hard to read acknowledged it was also "useful for determining the percentage of women affected in any particular country" and that it was "easy to understand at a glance, but harder to navigate" due to distortion, and that it was "misleading in the fact that countries' larger or smaller populations are not taken into account" (Participant 51). One comment that mentioned "easy to read" noted that the tile map made "it easy to understand the phenomenon proportions" (Participant 8). In response to what questions the maps evoked, a few participants asked about where and when the map types are used. Two responses about the tile maps stand out: "Where do people use the tile map? Where could that be useful?" (Participant 48) and "Who in the world would ever use tile maps? Yuk" (Participant 7).

Ten comments on the tile map mentioned the scissors icon that represented the data, and nine of these mentioned that in some way it was a good choice - that it conveyed the seriousness or emotional impact of the issue in some way or made the map more "interesting". For example, one said "the pictorial symbol, a scissor, helps to show the seriousness of the situation explicitly" (Participant 8) and another wrote "I like the scissors. A knife may have been even more effective, as a knife is more brutal" (Participant 23). Others commented ambiguously: "symbols may be less precise but may allow a directer (sic) connection to the displayed data (if chosen well)" (Participant 10). Conversely, one person noted that the tile map provided 
information well, but was geographically confusing and the scissor symbols were small and hard to see.

Comments that expressed discomfort or disdain for unfamiliar map type and country shapes evoke Huffman's (1997) critique of the limitations of the cognitive category and definitions of a Western map as ones that "make visible a global political world we recognize and understand" (p. 259), failing to recognize other images of the world as maps if they do not fit these familiar scientific or strict boundaries. These comments also come back to Crampton's (2004) critique of choropleth maps' popularity based on pre-defined geopolitical borders, which has in part spurred their popularity. While the thematic maps in this study did not overcome the notion of geopolitical borders due to the data available, they adapted the area that these boundaries form. The polygons in the cartogram were resized to be representative of national populations, and the polygons in the tile map were abstracted to a square so no country demanded more attention than another due to unequal size. Users' hesitancy towards the alternative geometries indicate that though these maps are not common place, they challenge users to perceive data, space, and visualization choices differently.

\section{Conclusion}

We produced and evaluated, through an online user survey, a choropleth, contiguous cartogram, and repeating icon tile map visualizing the SDG indicator data of the proportion of women and girls aged 15-49 who have undergone female genital mutilation/cutting. Users' comments and ratings of given map qualities show familiarity and acceptance for the choropleth map, but an admission that it is boring. The choropleth stimulated reflection on the usability of the map more than the design or the issue of $\mathrm{FGM} / \mathrm{C}$ while the scisssors symbol to represent $\mathrm{FGM} / \mathrm{C}$ rates in tile map was noted for conveying the seriousness of the data and issue, and for making the map more interesting. The comparative novelty and unfamiliarity of cartograms and tile maps may have confused or discouraged users, but the tile map showed highest performance on tasks. These maps also triggered attention to details such as symbolization, the phenomenon behind the numbers, and geometry. This preliminary investigation into the use of more than one thematic map type to represent SDG data suggests that there are many opportunities for future user research in cartography to emerging thematic map types representing social data by country on regional and global scales. In particular, future user studies can investigate how multiple and interactive thematic maps influence users' attitudes.

\section{References}

Blau, E. (2006). Isotype and Architecture in Red Vienna: The Modern Projects of Otto Neurath and Josef Frank. Austrian Studies, 14(Culture and Politics in Red Vienna), 227-259. Retrieved from http://www.jstor.org/stable/27944809

Cano, R. G., Buchin, K., Castermans, T., Pieterse, A., Sonke, W., \& Speckmann, B. (2015). Mosaic Drawings and Cartograms. Eurographics Conference on
Visualization (EuroVis), 34(3), 361-370. https://doi.org/10.1111/cgf.12648

Crampton, J. W. (2004). GIS and Geographic Governance: Reconstrucing the Choropleth Map. Cartographica, 39(1), 41-53. https://doi.org/10.3138/H066-3346-R9416382

Crampton, J. W. (2009). Rethinking Maps and Identity: Choropleths, Clines and Biopolitics Histories of critical mapping View project. https://doi.org/10.4324/9780203876848

DeBelius, D. (2015). Let's Tesselate: Hexagons For Tile Grid Maps | NPR Visuals. Retrieved April 6, 2018, from http://blog.apps.npr.org/2015/05/11/hex-tile-maps.html

Demers, S., \& Bortins, I. (2002). Cartogram Types. Retrieved August 13, 2018, from http://www.ncgia.ucsb.edu/projects/Cartogram_Central/ types.html

Dent, B. D. (1975). Communication Aspects of Value-byArea Cartograms. Cartography and Geographic Information Science, 2(2), 154-168. https://doi.org/10.1559/152304075784313278

Döll, P. (2017). Cartograms Facilitate Communication of Climate Change Risks and Responsibilities. Earth's Future, 5(12), 1182-1195. https://doi.org/10.1002/2017EF000677

Dörk, M., Feng, P., Collins, C., \& Carpendale, S. (2013). Critical InfoVis. In CHI ' 13 Extended Abstracts on Human Factors in Computing Systems on - CHI EA '13 (pp. 2189-2198). New York, New York, USA: ACM Press. https://doi.org/10.1145/2468356.2468739

Dorling, D. (1996). Area Cartograms: Their Use and Creation. https://doi.org/10.1002/9780470979587.ch33

Dorling, D., Barford, A., \& Newman, M. (2006). WORLDMAPPER: the world as you've never seen it before. IEEE Transactions on Visualization and Computer Graphics, 12(5), 757-764.

Dykes, J., MacEachren, A. M. \& Kraak, M. J. (2005). Introduction. Exploring geovisualization. 1st edn. Amsterdam: Elsevier.

Gastner, M. T., \& Newman, M. E. J. (2004). Diffusionbased method for producing density-equalizing maps. Proceedings of the National Academy of Sciences, 101(20), 7499-7504.

https://doi.org/10.1073/pnas.0400280101

Gilmartin, P. P. (1978). Evaluation of Thematic Maps Using the Semantic Differential Test. The American Cartographer, 5(2), 133-139. Retrieved from http://journals.sagepub.com/doi/10.1177/096466391246 7814

Haroz, S., Kosara, R., \& Franconeri, S. L. (2015). ISOTYPE Visualization - Working Memory, Performance, and Engagement with Pictographs. Proceedings of the 33rd Annual ACM Conference on Human Factors in Computing Systems - CHI '15, 11911200. https://doi.org/10.1145/2702123.2702275

Hennig, B. D., Pritchard, J., Ramsden, M., \& Dorling, D. (2010). Remapping the World's Population: Visualizing 
data as cartograms. ArcUser, Winter, 66-69. Retrieved from

http://www.esri.com/news/arcuser/0110/cartograms.htm 1

Huffman, N. H. (1997). Charting the Other Maps: Cartography and Visual Methods in Feminist Research. In J. P. I. Jones, H. J. Nast, \& S. M. Roberts (Eds.), Thresholds in Feminist Geography: Difference, Methodology, Representation (pp. 255-284). Rowman \& Littlefield Publishers, Inc.

Mapbox. (2018). Maki Icons | By Mapbox. Retrieved August 28, 2018, from https://www.mapbox.com/makiicons/

Mcneill, G., \& Hale, S. A. (2017). Generating Tile Maps. In Eurographics Conference on Visualization (EuroVis) 2017 (Vol. 36, pp. 435-445). https://doi.org/10.1111/cgf.13200

Monmonier, M. (1977). Maps, Distortion, and Meaning. Washington. Commission on College Geography Resource Paper 75-4. Association of American Geographers.

Monmonier, M. (1991). Ethics and map design. Six strategies for confronting the traditional one-map solution. Cartographic Perspectives, 10, 3-8. https://doi.org/10.14714/CP10.1052

Neurath, M. (1974). ISOTYPE. Instructural Science, 3, 127-150. Retrieved from https://link.springer.com/content/pdf/10.1007/BF00053 495.pdf

Nusrat, S., Alam, M. J., \& Kobourov, S. (2018). Evaluating Cartogram Effectiveness. IEEE Transactions on Visualization and Computer Graphics, 24(2), 11051118. https://doi.org/10.1109/TVCG.2016.2642109

Nusrat, S., \& Kobourov, S. (2015). Visualizing Cartograms: Goals and Task Taxonomy. Retrieved from http://arxiv.org/abs/1502.07792

Nusrat, S., \& Kobourov, S. (2016). The State of the Art in Cartograms. In Computer Graphics Forum (Vol. 35, pp. 619-642). https://doi.org/10.2312/eurovisstar.20141174

Olson, J. M. (1976). Noncontiguous area cartograms. The Professional Geographer, 28(4), 371-380.

ONE. (2018). ONE - Making the Connection. Retrieved April 22, 2018, from https://www.one.org/making-theconnection/index-en.html

Roser, M. (2018). Our World in Data. Retrieved April 8, 2018, from https://ourworldindata.org/

Roth, R. E., Woodruff, A. W., \& Johnson, Z. F. (2010). Value-by-alpha Maps: An Alternative Technique to the Cartogram. The Cartographic Journal, 47(2), 130-140. https://doi.org/10.1179/000870409X12488753453372

Schwabish, J. (2017). Tile Grid Waffle Chart Map in Excel. Retrieved August 24, 2018, from https://policyviz.com/2017/09/20/tile-grid-waffle-chartmap-in-excel/

Sui, D. Z., \& Holt, J. B. (2008). Visualizing and Analysing Public-Health Data Using Value-by-Area Cartograms:
Toward a New Synthetic Framework. Cartographica, 43(1), 3-20. https://doi.org/10.3138/carto.43.1.3

The World Bank. (2018). Visualize Inequality. Retrieved December 12, 2018 from http://www1.worldbank.org/poverty/visualizeinequality/

United Nations. (2018). The Sustainable Development Agenda. Retrieved May 26, 2018, from https://www.un.org/sustainabledevelopment/developme nt-agenda/

United Nations Statistics Division. (2018). Home - SDG Indicators. Retrieved May 26, 2018, from https://unstats.un.org/sdgs/

World Health Organization. (2008). Eliminating Female genital mutilation: An interagency statement. Retrieved 12 December, 2018 from https://www.who.int/reproductivehealth/publications/fg $\mathrm{m} / 9789241596442 / \mathrm{en} /$

Worldmapper. (2018). Worldmapper. Retrieved April 21, 2018, from https://worldmapper.org/ 